\title{
THE FINITE-TIME RUIN PROBABILITY OF THE COMPOUND POISSON MODEL WITH CONSTANT INTEREST FORCE
}

\author{
QIHE TANG, ${ }^{*}$ Concordia University
}

\begin{abstract}
In this paper, we establish a simple asymptotic formula for the finite-time ruin probability of the compound Poisson model with constant interest force and subexponential claims in the case that the initial surplus is large. The formula is consistent with known results for the ultimate ruin probability and, in particular, is uniform for all time horizons when the claim size distribution is regularly varying tailed.
\end{abstract}

Keywords: Asymptotics; finite-time ruin probability; Poisson process; regular variation; subexponentiality; uniform asymptotics; uniform convergence

2000 Mathematics Subject Classification: Primary 91B30

Secondary 60G70; 62P05

\section{The compound Poisson model}

Consider the compound Poisson model, in which the claim sizes $X_{k}, k=1,2, \ldots$, form a sequence of independent, identically distributed (i.i.d.), and nonnegative random variables with common distribution $B$, while the arrival times $\sigma_{k}, k=1,2, \ldots$, constitute a homogeneous Poisson process

$$
N(t)=\operatorname{card}\left\{k=1,2, \ldots: \sigma_{k} \leq t\right\}, \quad t \geq 0,
$$

with intensity $\lambda>0$. Let $\{C(t)\}_{t \geq 0}$, with $C(0)=0$, be a nondecreasing and right-continuous stochastic process denoting the total premium accumulated up to time $t$, let $r>0$ be the constant interest force (such that, after time $t$, one dollar becomes $\mathrm{e}^{r t}$ dollars), and let $x \geq 0$ be the initial surplus. Then the total surplus up to time $t$, denoted by $S_{r}(t)$, satisfies the equation

$$
S_{r}(t)=x \mathrm{e}^{r t}+\int_{0}^{t} \mathrm{e}^{r(t-s)} C(\mathrm{~d} s)-\sum_{k=1}^{N(t)} X_{k} \mathrm{e}^{r\left(t-\sigma_{k}\right)}, \quad t \geq 0,
$$

where, by convention, a summation over an empty set of indices is assumed to vanish.

As usual, we define the time to ruin of this model as

$$
\tau(x)=\inf \left\{t>0: S_{r}(t)<0 \mid S_{r}(0)=x\right\},
$$

where inf $\phi=\infty$ by convention. Hence, the probability of ruin within a finite time $T>0$ is defined by

$$
\psi_{r}(x, T)=\mathrm{P}(\tau(x) \leq T)
$$

Received 12 October 2004; revision received 22 January 2005.

* Postal address: Department of Mathematics and Statistics, Concordia University, 7141 Sherbrooke Street West, Montreal, Quebec H4B 1R6, Canada. Email address: qtang@ mathstat.concordia.ca 
while the probability of ultimate ruin is defined by

$$
\psi_{r}(x) \equiv \psi_{r}(x, \infty)=\lim _{T \rightarrow \infty} \psi_{r}(x, T)=\mathrm{P}(\tau(x)<\infty) .
$$

In this paper, we investigate the asymptotic behavior of the finite-time ruin probability $\psi_{r}(x, T)$, under the assumption that the claim size distribution $B$ is heavy tailed.

The remaining part of this paper consists of three sections. After briefly reviewing some related recent work in Section 2, we present two main results in Section 3 and prove them in Section 4 after preparing several lemmas.

\section{A brief review of related results}

Throughout, all limit relationships are for $x \rightarrow \infty$ unless stated otherwise, and, for two positive functions $a(\cdot)$ and $b(\cdot)$, we write $a(x) \sim b(x)$ if $\lim a(x) / b(x)=1$.

We shall restrict ourselves to the case of heavy-tailed claim size distributions. The most important class of heavy-tailed distributions is the subexponential class $\&$. By definition, a distribution $F$ on $[0, \infty)$ is subexponential, denoted by $F \in \delta$, if $\bar{F}(x)=1-F(x)>0$ for all $x \geq 0$ and

$$
\lim \frac{\overline{F^{* n}}(x)}{\bar{F}(x)}=n
$$

for some (and, hence, for all) $n=2,3, \ldots$, where $F^{* n}$ denotes the $n$-fold convolution of $F$; see Embrechts et al. (1979). It is well known that each subexponential distribution $F$ is long tailed, denoted by $F \in \mathcal{L}$, in the sense that the relation

$$
\lim \frac{\bar{F}(x+y)}{\bar{F}(x)}=1
$$

holds for each $y>0$. A useful subclass of subexponential distributions is $\mathcal{R}$, the class of distributions with regular variation. By definition, a distribution $F$ on $[0, \infty)$ belongs to the class $\mathcal{R}$ if $\bar{F}(x)>0$ for all $x \geq 0$ and there exists some $\alpha>0$ such that

$$
\lim \frac{\bar{F}(x y)}{\bar{F}(x)}=y^{-\alpha}
$$

for each $y>0$. We denote by $F \in \mathcal{R}_{-\alpha}$ the regularity property in (2.3). In this case, it is well known, for all $\alpha_{1}$ and $\alpha_{2}$ with $\alpha_{1}<\alpha<\alpha_{2}$, that

$$
\lim x^{\alpha_{1}} \bar{F}(x)=\lim \frac{1}{x^{\alpha_{2}} \bar{F}(x)}=0 ;
$$

hence, that the distribution $F$ has a finite moment of order $\alpha_{1}$. Furthermore, the asymptotic relation $\bar{F}(x y) \sim y^{-\alpha} \bar{F}(x)$, which is implied by (2.3), is automatically uniform for $y \in[a, b]$, with arbitrary, fixed $a$ and $b, 0<a \leq b<\infty$, in the sense that

$$
\lim \sup _{a \leq y \leq b}\left|\frac{\bar{F}(x y)}{y^{-\alpha} \bar{F}(x)}-1\right|=0 .
$$

For more details of heavy-tailed distributions and their applications to insurance and finance, the reader is referred to Bingham et al. (1987) and Embrechts et al. (1997). 
The asymptotic behavior of the ultimate ruin probability $\psi_{r}(x)$ of the risk model introduced in Section 1 - with $C(\cdot)$ a deterministic linear function, $B$ heavy tailed, and $\left\{X_{k}\right\}_{k=1}^{\infty}$ and $\{N(t)\}_{t \geq 0}$ mutually independent - has been investigated in the recent literature. Under the condition $B \in \mathcal{R}_{-\alpha}$ for some $\alpha>1$, starting from an integral equation of Sundt and Teugels (1995), Klüppelberg and Stadtmüller (1998) developed a sophisticated $L_{p}$-transform technique in proving the result

$$
\psi_{r}(x) \sim \frac{\lambda}{\alpha r} \bar{B}(x)
$$

see their Corollary 2.4. Asmussen (1998, Corollary 4.1(ii)) and Asmussen et al. (2002) proved a more general result, namely that the relation

$$
\psi_{r}(x) \sim \frac{\lambda}{r} \int_{x}^{\infty} \frac{\bar{B}(y)}{y} \mathrm{~d} y
$$

holds under the condition $B \in 8^{*}$, where the class $8^{*}$ was introduced by Klüppelberg (1988) and is characterized by the relation

$$
\int_{0}^{x} \bar{B}(x-y) \bar{B}(y) \mathrm{d} y \sim 2 \mu \bar{B}(x),
$$

with $\mu=\int_{0}^{\infty} \bar{B}(y) \mathrm{d} y \in(0, \infty)$. Klüppelberg (1988, Theorem 3.2) pointed out that if $B \in 8^{*}$ then both $B$ and its integrated tail distribution $B_{\mathrm{I}}$, which is defined by

$$
B_{\mathrm{I}}(x)=\frac{1}{\mu} \int_{0}^{x} \bar{B}(y) \mathrm{d} y, \quad x \geq 0,
$$

are subexponential. Lately, also based on the work of Sundt and Teugels (1995), but using a simpler treatment, Kalashnikov and Konstantinides (2000) and Konstantinides et al. (2002) rederived (2.6) under the condition that the integrated tail distribution $B_{\mathrm{I}}$ is an element of the class $\mathcal{A}$; that is, $B_{\mathrm{I}}$ is subexponential and satisfies

$$
\limsup \frac{\overline{B_{\mathrm{I}}}(x y)}{\overline{B_{\mathrm{I}}}(x)}<1 \quad \text { for some } y>1 .
$$

To the author's knowledge, whether the condition $B_{\mathrm{I}} \in \delta$ is sufficient to establish (2.6) remains unknown.

It is also worth mentioning that $B \in 8^{*}$ neither implies nor is implied by $B_{\mathrm{I}} \in \mathcal{A}$. A simple illustration of the assertion of the lack of sufficiency (i.e. $B \in \mathcal{f}^{*} \nRightarrow B_{\mathrm{I}} \in \mathcal{A}$ ) is a distribution with a tail satisfying

$$
\bar{B}(x) \sim x^{-1} \ln ^{-2} x .
$$

To illustrate the lack of necessity (i.e. $B \in 8^{*} \nLeftarrow B_{\mathrm{I}} \in \mathcal{A}$ ), let us look at the random variable

$$
Z=a^{\pi}
$$

where $\pi$ is geometric with probability function $\mathrm{P}(\pi=k)=(1-p) p^{k}, 0<p<1$, $k=0,1, \ldots$, and $a$ is arbitrarily fixed and satisfies $1<a<1 / p$. Clearly, the random variable $Z$ has a finite mean and its distribution $B$ satisfies

$$
\lim \frac{\bar{B}(a x)}{\bar{B}(x)}=p<\infty \text {. }
$$


Based on this, it is easy to see that $B_{\mathrm{I}} \in \delta$ (see Theorem 1 of Embrechts and Omey (1984) or Proposition 1.4.4 of Embrechts et al. (1997)), that $B \notin \mathcal{L}$ (and, hence, $B \notin 8^{*}$ ), and that

$$
\lim \frac{\overline{B_{\mathrm{I}}}(a x)}{\overline{B_{\mathrm{I}}}(x)}=a p<1 .
$$

Therefore, $B_{\mathrm{I}} \in \mathcal{A}$.

Note that, for an arbitrarily large number $v>0$, by choosing the parameters $a$ and $p$ in (2.7) so that $a^{v} p<1$, we have $\mathrm{E} Z^{v}<\infty$. This means that the condition $B_{\mathrm{I}} \in \mathcal{A}$ allows for some distributions that are not so 'heavy tailed' and are not in the class $\mathcal{L}$ (and, hence, are not in the class $\left.8^{*}\right)$.

Recently, Tang (2004) extended the work of Konstantinides et al. (2002) to the discrete-time model, while Tang (2005) extended the work of Klüppelberg and Stadtmüller (1998) to the ordinary renewal model.

\section{Main results}

In this paper, we use a different method to establish a similar formula for the finite-time ruin probability, with $B$ ranging over the whole class $\&$. Our first main result is the following theorem.

Theorem 3.1. Consider the compound Poisson model introduced in Section 1, in which all sources of randomness, namely $\left\{X_{k}\right\}_{k=1}^{\infty},\{N(t)\}_{t \geq 0}$, and $\{C(t)\}_{t \geq 0}$, are mutually independent. If $B \in \&$ then, for each $T>0$,

$$
\psi_{r}(x, T) \sim \frac{\lambda}{r} \int_{x}^{x \mathrm{e}^{r T}} \frac{\bar{B}(y)}{y} \mathrm{~d} y .
$$

Note that (3.1) is consistent with (2.6). In particular, if $B \in \mathcal{R}_{-\alpha}$ for some $\alpha>0$ then, by the local uniformity property $(2.4)$, we have

$$
\begin{aligned}
\int_{x}^{x \mathrm{e}^{r T} \frac{\bar{B}}{(y)}} \frac{\mathrm{d} y}{y} & =\bar{B}(x) \int_{x}^{x \mathrm{e}^{r T}} \frac{\bar{B}(y)}{\bar{B}(x)} \frac{1}{y} \mathrm{~d} y \\
& \sim \bar{B}(x) \int_{x}^{x \mathrm{e}^{r T}}\left(\frac{y}{x}\right)^{-\alpha} \frac{1}{y} \mathrm{~d} y \\
& =\frac{1}{\alpha} \bar{B}(x)\left(1-\mathrm{e}^{-\alpha r T}\right) .
\end{aligned}
$$

Hence, in this case, it follows from (3.1) that, for each $T>0$,

$$
\psi_{r}(x, T) \sim \frac{\lambda}{\alpha r} \bar{B}(x)\left(1-\mathrm{e}^{-\alpha r T}\right),
$$

which is consistent with (2.5).

For each $T \in(0, \infty]$, we denote by $\widetilde{C}(T)$ the total discounted premium accumulated up to time $T$, that is

$$
\widetilde{C}(T)=\int_{0}^{T} \mathrm{e}^{-r t} C(\mathrm{~d} t) \text { for } T \in(0, \infty] .
$$


The following result makes the statement of (3.2) somewhat stronger.

Theorem 3.2. Consider the compound Poisson model introduced in Section 1 , with $B \in \mathcal{R}_{-\alpha}$ for some $\alpha>0$ and $\widetilde{C}(\infty)$ in (3.3) almost surely finite. Then (3.2) holds uniformly for $T \in(0, \infty]$, i.e.

$$
\lim \sup _{0<T \leq \infty}\left|\frac{\psi_{r}(x, T)}{(\lambda / \alpha r) \bar{B}(x)\left(1-\mathrm{e}^{-\alpha r T}\right)}-1\right|=0,
$$

if either

1. $\left\{X_{k}\right\}_{k=1}^{\infty},\{N(t)\}_{t \geq 0}$, and $\{C(t)\}_{t \geq 0}$ are mutually independent, or

2. $\left\{X_{k}\right\}_{k=1}^{\infty}$ and $\{N(t)\}_{t \geq 0}$ are mutually independent and $\{\widetilde{C}(\infty)\}_{T \in(0, \infty]}$ satisfies

$$
\mathrm{P}(\widetilde{C}(\infty)>x)=o(\bar{B}(x)) \text { and } \quad \lim \sup _{0 \leq T \leq 1} \frac{\mathrm{P}(\widetilde{C}(T)>x)}{T \bar{B}(x)}=0 .
$$

As pointed out by Tang (2005), allowing dependence between the premium process and the claim process is not only of purely academic interest since the premium rate very often depends on the history of the surplus process.

Admittedly, there are a lot of advantages in knowing the uniformity of an asymptotic relation. Below are some direct applications of the uniformity described by Theorem 3.2.

(I) The relation

$$
\psi_{r}(x, T(x)) \sim \frac{\lambda}{\alpha r} \bar{B}(x)\left(1-\mathrm{e}^{-\alpha r T(x)}\right)
$$

holds for every function $T(\cdot) \in(0, \infty]$. Moreover, if $T(x) \rightarrow \infty$ then the relation above reduces to

$$
\psi_{r}(x, T(x)) \sim \frac{\lambda}{\alpha r} \bar{B}(x) \sim \psi_{r}(x) .
$$

(II) For a random variable $\mathcal{T}$, which is independent of the risk system and has a distribution $H$ with $\bar{H}(0)>0$, denote by $\psi_{r}(x, \mathcal{T})$ the probability of 'ruin within the random horizon $\mathcal{T}$ '. We have

$$
\begin{aligned}
\psi_{r}(x, \mathcal{T}) & =\int_{0}^{\infty} \psi_{r}(x, T) H(\mathrm{~d} T) \\
& \sim \int_{0}^{\infty} \frac{\lambda}{\alpha r} \bar{B}(x)\left(1-\mathrm{e}^{-\alpha r T}\right) H(\mathrm{~d} T) \\
& =\frac{\lambda}{\alpha r} \bar{B}(x) \mathrm{E}\left(1-\mathrm{e}^{-\alpha r \mathcal{T}}\right) \mathbf{1}_{\{\mathcal{T}>0\}},
\end{aligned}
$$

where $\mathbf{1}_{A}$ denotes the indicator function of an event $A$.

(III) Relation (3.5) further enables us to derive an asymptotic estimate for the Laplace-Stieltjes transform of the ruin time $\tau(x)$. To this end, we take $\mathcal{T}$ in (3.5) to be an exponentially distributed random variable with mean $1 / \kappa$. On the one hand, recalling (1.3) and using Fubini's theorem, we have

$$
\psi_{r}(x, \mathcal{T})=\int_{0}^{\infty} \mathrm{E} \mathbf{1}_{\{\tau(x) \leq T\}} H(\mathrm{~d} T)=\mathrm{Ee}^{-\kappa \tau(x)} \mathbf{1}_{\{\tau(x)<\infty\}}=\mathrm{Ee}^{-\kappa \tau(x)}
$$


on the other hand, (3.5) gives

$$
\psi_{r}(x, \mathcal{T}) \sim \frac{\lambda}{\alpha r} \bar{B}(x) \mathrm{E}\left(1-\mathrm{e}^{-\alpha r \mathcal{T}}\right)=\frac{\lambda}{\alpha r+\kappa} \bar{B}(x) .
$$

It follows that

$$
\mathrm{Ee}^{-\kappa \tau(x)} \sim \frac{\lambda}{\alpha r+\kappa} \bar{B}(x)
$$

\section{Proofs of Theorems 3.1 and 3.2}

\subsection{Lemmas}

Before giving the proofs we must establish some preliminary results.

Lemma 4.1. If $F$ is subexponential then, for each $\varepsilon>0$, there exists some constant $C_{\varepsilon}>0$ such that the inequality

$$
\overline{F^{* n}}(x) \leq C_{\varepsilon}(1+\varepsilon)^{n} \bar{F}(x)
$$

holds for all $n=1,2, \ldots$ and $x \geq 0$.

Proof. This inequality is well known and was established by Chistyakov (1964) and Athreya and Ney (1972); see also Embrechts et al. (1997, Lemma 1.3.5).

Lemma 4.2. Let $X$ and $Y$ be two independent and nonnegative random variables. If $X$ is subexponentially distributed while $Y$ is bounded and nondegenerate at 0 , then the product $X Y$ is subexponentially distributed.

Proof. See Corollary 2.5 of Cline and Samorodnitsky (1994).

Lemma 4.3. Let $\{N(t)\}_{t \geq 0}$ be a Poisson process with arrival times $\sigma_{k}, k=1,2, \ldots$ Given $N(T)=n$ for arbitrarily fixed $T>0$ and $n=1,2, \ldots$, the random vector $\left(\sigma_{1}, \ldots, \sigma_{n}\right)$ is equal in distribution to the random vector $\left(T U_{(1, n)}, \ldots, T U_{(n, n)}\right)$, where $U_{(1, n)}, \ldots, U_{(n, n)}$ denote the order statistics of $n$ random variables, $U_{1}, \ldots, U_{n}$, independent and identically uniformly distributed on $(0,1)$.

Proof. This result is also well known; see, for example, Theorem 2.3.1 of Ross (1983).

Lemma 4.4. If a sequence of distributions $\left\{F_{t}\right\}_{t \geq 0}$ converges to a continuous distribution $F$ as $t \rightarrow \infty$, then the convergence is uniform in the sense that

$$
\lim _{t \rightarrow \infty} \sup _{-\infty \leq x \leq \infty}\left|F_{t}(x)-F(x)\right|=0 .
$$

Proof. See Theorem 1.11 of Petrov (1995) (where the sequence under discussion is in fact $\left\{F_{n}\right\}_{n=1}^{\infty}$ instead of $\left\{F_{t}\right\}_{t \geq 0}$.

The following result may be interesting in its own right.

Lemma 4.5. Let $\left\{X_{k}\right\}_{k=1}^{\infty}$ be a sequence of i.i.d. random variables with common distribution $B \in \mathcal{R}_{-\alpha}$ for some $\alpha>0$; let $\left\{V_{k}\right\}_{k=1}^{\infty}$ be another sequence of identically distributed (not necessarily independent) and bounded random variables; let $\{N(t)\}_{t \geq 0}$ be a Poisson process with intensity $\lambda>0$; and let these sources of randomness be mutually independent. Then, for arbitrary, fixed $T$, uniformly for $t, 0<t \leq T$, we have

$$
\mathrm{P}\left(\sum_{k=1}^{N(t)} X_{k} \mathrm{e}^{-t V_{k}}>x\right) \sim \lambda t \mathrm{P}\left(X_{1} \mathrm{e}^{-t V_{1}}>x\right) \sim \lambda t \mathrm{E}^{-\alpha t V_{1}} \bar{B}(x) .
$$


Proof. Suppose that the random variable $V_{1}$ is distributed by $G$ with finite support within $(-M, M)$, for some $M>0$. The last relation in (4.1) is immediate from the local uniformity property (2.4) as, uniformly for $t, 0<t \leq T$, we have

$$
\mathrm{P}\left(X_{1} \mathrm{e}^{-t V_{1}}>x\right)=\bar{B}(x) \int_{-M}^{M} \frac{\bar{B}\left(\mathrm{e}^{t v} x\right)}{\bar{B}(x)} G(\mathrm{~d} v) \sim \bar{B}(x) \int_{-M}^{M} \mathrm{e}^{-\alpha t v} G(\mathrm{~d} v) .
$$

Let us now prove the first relation in (4.1). For a temporarily fixed positive integer $N$, we find that

$$
\begin{aligned}
\mathrm{P}\left(\sum_{k=1}^{N(t)} X_{k} \mathrm{e}^{-t V_{k}}>x\right) & =\left(\sum_{n=1}^{N}+\sum_{n=N+1}^{\infty}\right) \mathrm{P}\left(\sum_{k=1}^{n} X_{k} \mathrm{e}^{-t V_{k}}>x\right) \mathrm{P}(N(t)=n) \\
& =: I_{1}(x, t, N)+I_{2}(x, t, N) .
\end{aligned}
$$

Consider $I_{1}(x, t, N)$. We apply Proposition 5.1 of Tang and Tsitsiashvili (2003), which says that, for i.i.d. subexponential random variables $\left\{X_{k}\right\}_{k=1}^{N}$ and for arbitrarily fixed $a$ and $b$, $0<a \leq b<\infty$, the relation

$$
\mathrm{P}\left(\sum_{k=1}^{N} c_{k} X_{k}>x\right) \sim \sum_{k=1}^{N} \mathrm{P}\left(c_{k} X_{k}>x\right)
$$

holds uniformly for $\left(c_{1}, \ldots, c_{N}\right) \in[a, b] \times \cdots \times[a, b]$. Hence, in this situation, by conditioning on $\left(V_{1}, \ldots, V_{N}\right)$ we find that

$$
I_{1}(x, t, N) \sim \mathrm{P}\left(X_{1} \mathrm{e}^{-t V_{1}}>x\right) \sum_{n=1}^{N} n \mathrm{P}(N(t)=n)
$$

holds uniformly for $t, 0<t \leq T$. Furthermore, it is clear that, for each $v>0$,

$$
\lim _{N \rightarrow \infty} \sup _{0<t \leq T} \frac{1}{\lambda t} \mathrm{E}(N(t))^{v} \mathbf{1}_{\{N(t)>N\}}=0 .
$$

It follows, from (4.3) and (4.4), that

$$
\lim _{N \rightarrow \infty} \lim \sup _{0<t \leq T}\left|\frac{I_{1}(x, t, N)}{\lambda t \mathrm{P}\left(X_{1} \mathrm{e}^{-t V_{1}}>x\right)}-1\right|=\lim _{N \rightarrow \infty} \sup _{0<t \leq T} \frac{1}{\lambda t} \mathrm{E} N(t) \mathbf{1}_{\{N(t)>N\}}=0 .
$$

Now consider $I_{2}(x, t, N)$. We first state the following probabilistic inequality, which can easily be verified using Theorem 1.1 of Nagaev (1979): for a sequence of i.i.d. random variables $\left\{X_{k}\right\}_{k=1}^{\infty}$, with common distribution $B$ and finite moment of order $\kappa \in(0,1)$, the inequality

$$
\mathrm{P}\left(\sum_{k=1}^{n} X_{k}>x\right) \leq n \bar{B}\left(\frac{x}{v}\right)+\left(\frac{\mathrm{e} \mathrm{E} X_{1}^{\kappa}}{v^{1-\kappa}}\right)^{v} n^{v} x^{-v \kappa}
$$

holds for all $n=1,2, \ldots, x>0$, and $v>0$. For $B \in \mathcal{R}_{-\alpha}$, by choosing some $\kappa \in$ $(0, \min \{1, \alpha\})$ and $v>\alpha / \kappa>1$, we find that

$$
\mathrm{P}\left(\sum_{k=1}^{n} X_{k}>x\right) \leq C_{v} n^{v} \bar{B}(x)
$$


for some $C_{v}>0$, all $n=1,2, \ldots$, and all $x>0$. Hence, as $x \rightarrow \infty$,

$$
\begin{aligned}
I_{2}(x, t, N) & \leq \sum_{n=N+1}^{\infty} \mathrm{P}\left(\sum_{k=1}^{n} X_{k}>x \mathrm{e}^{-T M}\right) \mathrm{P}(N(t)=n) \\
& \leq C_{v} \bar{B}\left(x \mathrm{e}^{-T M}\right) \mathrm{E}(N(t))^{v} \mathbf{1}_{\{N(t)>N\}} \\
& \sim C_{v} \mathrm{e}^{2 \alpha T M} \bar{B}\left(x \mathrm{e}^{T M}\right) \mathrm{E}(N(t))^{v} \mathbf{1}_{\{N(t)>N\}} \\
& \leq C_{v} \mathrm{e}^{2 \alpha T M} \mathrm{P}\left(X_{1} \mathrm{e}^{-t V_{1}}>x\right) \mathrm{E}(N(t))^{v} \mathbf{1}_{\{N(t)>N\}} .
\end{aligned}
$$

It follows, from (4.6) and (4.4), that

$$
\lim _{N \rightarrow \infty} \lim \sup \sup _{0<t \leq T} \frac{I_{2}(x, t, N)}{\lambda t \mathrm{P}\left(X_{1} \mathrm{e}^{-t V_{1}}>x\right)}=0 .
$$

We conclude, from (4.2), (4.5), and (4.7), that the first relation in (4.1) holds uniformly for $t$, $0<t \leq T$. This ends the proof of Lemma 4.5.

\subsection{Proof of Theorem 3.1}

It follows, from (1.3) and (1.2), that

$$
\psi_{r}(x, T)=\mathrm{P}\left(\mathrm{e}^{-r t} S_{r}(t)<0 \text { for some } t \in(0, T] \mid S_{r}(0)=x\right) .
$$

Furthermore, for each $t \in(0, T]$, it follows from (1.1) that

$$
x-\sum_{k=1}^{N(T)} X_{k} \mathrm{e}^{-r \sigma_{k}} \leq \mathrm{e}^{-r t} S_{r}(t) \leq x+\widetilde{C}(T)-\sum_{k=1}^{N(t)} X_{k} \mathrm{e}^{-r \sigma_{k}},
$$

where $\widetilde{C}(T)$ is as defined in (3.3). For notational convenience, we write $\widetilde{X}(t)=\sum_{k=1}^{N(t)} X_{k} \mathrm{e}^{-r \sigma_{k}}$ for the total discounted claim amount accumulated up to time $t>0$. Clearly, (4.8) and the first inequality in (4.9) imply that

$$
\psi_{r}(x, T) \leq \mathrm{P}(\tilde{X}(T)>x),
$$

while (4.8) and the second inequality in (4.9) imply that

$$
\psi_{r}(x, T) \geq \mathrm{P}(\tilde{X}(t)>x+\widetilde{C}(T) \text { for some } t \in(0, T])=\mathrm{P}(\tilde{X}(T)>x+\widetilde{C}(T)) .
$$

Hence, if we prove that

$$
\mathrm{P}(\tilde{X}(T)>x+\widetilde{C}(T)) \sim \mathrm{P}(\tilde{X}(T)>x) \sim \lambda \int_{0}^{T} \mathrm{P}\left(X_{1} \mathrm{e}^{-r u}>x\right) \mathrm{d} u,
$$

then it follows that

$$
\psi_{r}(x, T) \sim \lambda \int_{0}^{T} \mathrm{P}\left(X_{1} \mathrm{e}^{-r u}>x\right) \mathrm{d} u,
$$

which, upon a trivial substitution, implies the required result (3.1). 
Thus, let us prove the two asymptotic relations in (4.12). By Lemma 4.3, we have

$$
\begin{aligned}
\mathrm{P}(\tilde{X}(T)>x) & =\sum_{n=1}^{\infty} \mathrm{P}\left(\sum_{k=1}^{n} X_{k} \mathrm{e}^{-r \sigma_{k}}>x \mid N(T)=n\right) \mathrm{P}(N(T)=n) \\
& =\sum_{n=1}^{\infty} \mathrm{P}\left(\sum_{k=1}^{n} X_{k} \mathrm{e}^{-r T U_{(k, n)}}>x\right) \mathrm{P}(N(T)=n),
\end{aligned}
$$

where $U_{(k, n)}$, for $k=1,2, \ldots, n$ and $n=1,2, \ldots$, are as in Lemma 4.3 and are independent of $\left\{X_{k}\right\}_{k=1}^{\infty}$. Therefore,

$$
\mathrm{P}(\tilde{X}(T)>x)=\sum_{n=1}^{\infty} \mathrm{P}\left(\sum_{k=1}^{n} X_{k} \mathrm{e}^{-r T U_{k}}>x\right) \mathrm{P}(N(T)=n) .
$$

By Lemma 4.2, we know that the i.i.d. products $X_{k} \mathrm{e}^{-r T U_{k}}, k=1,2, \ldots$, are subexponentially distributed; by Lemma 4.1 we also know that for an arbitrarily fixed $\varepsilon>0$, there exists a constant $C_{\varepsilon}>0$ such that the inequality

$$
\mathrm{P}\left(\sum_{k=1}^{n} X_{k} \mathrm{e}^{-r T U_{k}}>x\right) \leq C_{\varepsilon}(1+\varepsilon)^{n} \mathrm{P}\left(X_{1} \mathrm{e}^{-r T U_{1}}>x\right)
$$

holds for all $n=1,2, \ldots$ and $x \geq 0$. Since $\mathrm{E}(1+\varepsilon)^{N(T)}<\infty$, by applying both the definition (in (2.1)) of subexponentiality and the dominated convergence theorem, we find, from (4.13), that

$$
\begin{aligned}
\mathrm{P}(\tilde{X}(T)>x) & \sim \mathrm{P}\left(X_{1} \mathrm{e}^{-r T U_{1}}>x\right) \sum_{n=1}^{\infty} n \mathrm{P}(N(T)=n) \\
& =\lambda \int_{0}^{T} \mathrm{P}\left(X_{1} \mathrm{e}^{-r u}>x\right) \mathrm{d} u .
\end{aligned}
$$

This proves the second relation in (4.12).

Using (4.14), it is not difficult to prove the first asymptotic relation in (4.12). In fact, since the product $X_{1} \mathrm{e}^{-r T U_{1}}$ is subexponentially distributed, by (4.14) it is easy to see that the sum $\widetilde{X}(T)$ is long tailed. Using the dominated convergence theorem and property (2.2) of long-tailed distributions, we find that

$$
\lim \frac{\mathrm{P}(\tilde{X}(T)>x+\widetilde{C}(T))}{\mathrm{P}(\widetilde{X}(T)>x)}=\int_{[0, \infty)} \lim \frac{\mathrm{P}(\tilde{X}(T)>x+y)}{\mathrm{P}(\widetilde{X}(T)>x)} \mathrm{P}(\widetilde{C}(T) \in \mathrm{d} y)=1 .
$$

This ends the proof of Theorem 3.1.

\subsection{Proof of Theorem 3.2}

First, we prove that (3.2) holds for each $T \in(0, \infty]$. Since, under both assumptions, the relation

$$
\psi_{r}(x) \sim \frac{\lambda}{\alpha r} \bar{B}(x)
$$

is a direct consequence of Theorem 1 of Tang (2005), and since, under assumption 1 of Theorem 3.2, (3.2) with $T \in(0, \infty)$ follows from Theorem 3.1, we need only prove (3.2) 
for each $T \in(0, \infty)$ under assumption 2 of Theorem 3.2. In this case, following the proof of Theorem 3.1, inequalities (4.10) and (4.11) remain valid and, moreover,

$$
\mathrm{P}(\tilde{X}(T)>x) \sim \lambda \int_{0}^{T} \mathrm{P}\left(X_{1} \mathrm{e}^{-r u}>x\right) \mathrm{d} u \sim \frac{\lambda}{\alpha r} \bar{B}(x)\left(1-\mathrm{e}^{-\alpha r T}\right) .
$$

Hence, it suffices to prove that

$$
\liminf \frac{\mathrm{P}(\tilde{X}(T)>x+\widetilde{C}(T))}{\mathrm{P}(\widetilde{X}(T)>x)} \geq 1 .
$$

To this end, note that (4.16) indicates that the distribution of $\widetilde{X}(T)$ belongs to the class $\mathcal{R}_{-\alpha}$. For arbitrary, fixed $l>0$, by applying (4.16) and (3.4) we find that

$$
\begin{aligned}
& \lim \inf \frac{\mathrm{P}(\tilde{X}(T)>x+\widetilde{C}(T))}{\mathrm{P}(\widetilde{X}(T)>x)} \\
& \quad \geq \liminf \frac{\mathrm{P}(\tilde{X}(T)>(1+l) x)-\mathrm{P}(\widetilde{C}(\infty)>l x)}{\mathrm{P}(\widetilde{X}(T)>x)} \\
& \quad \geq \liminf \frac{\mathrm{P}(\tilde{X}(T)>(1+l) x)}{\mathrm{P}(\widetilde{X}(T)>x)}-\lim \sup \frac{\mathrm{P}(\widetilde{C}(\infty)>l x)}{\bar{B}(l x)} \frac{\bar{B}(l x)}{\bar{B}(x)} \frac{\bar{B}(x)}{(\lambda / \alpha r) \bar{B}(x)\left(1-\mathrm{e}^{-\alpha r T}\right)} \\
& \quad=(1+l)^{-\alpha} .
\end{aligned}
$$

Hence, (4.17) follows since $l$ can be chosen arbitrarily close to 0 .

Second, we prove the uniformity of (3.2) for $T \in(1, \infty]$. Write $\mathrm{P}^{(x)}(\cdot)=\mathrm{P}(\cdot \mid \tau(x)<\infty)$, for $x \geq 0$, and recall the definition in (1.3). From (3.2) and (4.15) we find that

$$
\lim \mathrm{P}^{(x)}(\tau(x) \leq T)=\lim \frac{\psi_{r}(x, T)}{\psi_{r}(x)}=1-\mathrm{e}^{-\alpha r T}
$$

for each $T \in(0, \infty]$. This means that in $\mathrm{P}^{(x)}$, the limit distribution of the ruin time $\tau(x)$ is exponential with mean $1 / \alpha r$. By applying Lemma 4.4, we see that the convergence in (4.19) is uniform with respect to $T \in(0, \infty]$, that is

$$
\lim \sup _{0<T \leq \infty}\left|\frac{\psi_{r}(x, T)}{\psi_{r}(x)}-\left(1-\mathrm{e}^{-\alpha r T}\right)\right|=0 .
$$

Using (4.15), we can easily prove that (3.2) holds uniformly for $T \in(1, \infty]$.

Finally, to end the proof of Theorem 3.2, it remains to prove the uniformity of (3.2) for $T \in(0,1]$. By (4.13) and Lemma 4.5, we have

$$
\mathrm{P}(\tilde{X}(T)>x) \sim \frac{\lambda}{\alpha r} \bar{B}(x)\left(1-\mathrm{e}^{-\alpha r T}\right)
$$

uniformly for $T \in(0,1]$. It follows, from this and (4.10), that

$$
\limsup \sup _{0<T \leq 1} \frac{\psi_{r}(x, T)}{(\lambda / \alpha r) \bar{B}(x)\left(1-\mathrm{e}^{-\alpha r T}\right)} \leq 1 .
$$

Using (4.11) and Lemma 4.5, we can also prove that

$$
\liminf \inf _{0<T \leq 1} \frac{\psi_{r}(x, T)}{(\lambda / \alpha r) \bar{B}(x)\left(1-\mathrm{e}^{-\alpha r T}\right)} \geq 1 .
$$


In fact, under assumption 1 of Theorem 3.2, by using (4.11), (4.20), and Fatou's lemma in turn, we obtain

$$
\begin{aligned}
& \lim \inf \inf _{0<T \leq 1} \frac{\psi_{r}(x, T)}{(\lambda / \alpha r) \bar{B}(x)\left(1-\mathrm{e}^{-\alpha r T}\right)} \geq \liminf \inf _{0<T \leq 1} \frac{\mathrm{P}(\tilde{X}(T)>x+\widetilde{C}(1))}{(\lambda / \alpha r) \bar{B}(x)\left(1-\mathrm{e}^{-\alpha r T}\right)} \\
& \quad=\liminf \inf _{0<T \leq 1} \int_{[0, \infty)} \frac{(\lambda / \alpha r) \bar{B}(x+y)\left(1-\mathrm{e}^{-\alpha r T}\right)}{(\lambda / \alpha r) \bar{B}(x)\left(1-\mathrm{e}^{-\alpha r T}\right)} \mathrm{P}(\widetilde{C}(1) \in \mathrm{d} y) \\
& \quad \geq \int_{[0, \infty)} \liminf \frac{\bar{B}(x+y)}{\bar{B}(x)} \mathrm{P}(\widetilde{C}(1) \in \mathrm{d} y) \\
&=1 .
\end{aligned}
$$

Under assumption 2 of Theorem 3.2, and analogous to (4.18), by using (4.11), (4.20), and (3.4) we find, for arbitrary, fixed $l>0$, that

$$
\begin{aligned}
\liminf \inf _{0<T \leq 1} \frac{\psi_{r}(x, T)}{(\lambda / \alpha r) \bar{B}(x)\left(1-\mathrm{e}^{-\alpha r T}\right)} & \geq \liminf \inf _{0<T \leq 1} \frac{\mathrm{P}(\tilde{X}(T)>(1+l) x)-\mathrm{P}(\widetilde{C}(T)>l x)}{(\lambda / \alpha r) \bar{B}(x)\left(1-\mathrm{e}^{-\alpha r T}\right)} \\
& =\liminf \inf _{0<T \leq 1} \frac{(\lambda / \alpha r) \bar{B}((1+l) x)\left(1-\mathrm{e}^{-\alpha r T}\right)}{(\lambda / \alpha r) \bar{B}(x)\left(1-\mathrm{e}^{-\alpha r T}\right)} \\
& =(1+l)^{-\alpha} .
\end{aligned}
$$

Thus, (4.21) holds under both assumptions. This ends the proof of Theorem 3.2.

\section{Acknowledgements}

The author is grateful for the referee's thoughtful comments on the study of asymptotic ruin probabilities and to Professor Ralph P. Russo at the University of Iowa and Ms Yu Chen at the University of Science and Technology of China for their stimulating discussions. This work was supported by the Natural Sciences and Engineering Research Council of Canada (grant no. 311990) and the National Natural Science Foundation of China (grant no. 70471071).

\section{References}

Asmussen, S. (1998). Subexponential asymptotics for stochastic processes: extremal behavior, stationary distributions and first passage probabilities. Ann. Appl. Prob. 8, 354-374.

Asmussen, S. et al. (2002). A local limit theorem for random walk maxima with heavy tails. Statist. Prob. Lett. 56, 399-404.

Athreya, K. B. AND Ney, P. E. (1972). Branching Processes. Springer, New York.

Bingham, N. H., Goldie, C. M. And Teugels, J. L. (1987). Regular Variation. Cambridge University Press.

Chistyakov, V.P. (1964). A theorem on sums of independent positive random variables and its applications to branching random processes. Teor. Verojatnost. i Primenen 9, 710-718 (in Russian). English translation: Theory Prob. Appl. 9, 640-648.

Cline, D. B. H. and SAmorodnitsky, G. (1994). Subexponentiality of the product of independent random variables. Stoch. Process. Appl. 49, 75-98.

Embrechts, P. And Omey, E. (1984). A property of longtailed distributions. J. Appl. Prob. 21, 80-87.

Embrechts, P., Goldie, C. M. and VeraverbeKe, N. (1979). Subexponentiality and infinite divisibility. Z. Wahrscheinlichkeitsth. 49, 335-347.

Embrechts, P., Klüppelberg, C. AND Mikosch, T. (1997). Modelling Extremal Events for Insurance and Finance. Springer, Berlin.

Kalashnikov, V. and Konstantinides, D. (2000). Ruin under interest force and subexponential claims: a simple treatment. Insurance Math. Econom. 27, 145-149.

KLÜPPElberg, C. (1988). Subexponential distributions and integrated tails. J. Appl. Prob. 25, 132-141. 
Klüppelberg, C. And Stadtmüller, U. (1998). Ruin probabilities in the presence of heavy-tails and interest rates. Scand. Actuarial J. 1998, 49-58.

Konstantinides, D., Tang, Q. And Tsitsiashvili, G. (2002). Estimates for the ruin probability in the classical risk model with constant interest force in the presence of heavy tails. Insurance Math. Econom. 31, 447-460.

Nagaev, S. V. (1979). Large deviations of sums of independent random variables. Ann. Prob. 7, 745-789.

Petrov, V. V. (1995). Limit Theorems of Probability Theory. Sequences of Independent Random Variables. Oxford University Press.

Ross, S. M. (1983). Stochastic Processes. John Wiley, New York.

Sundt, B. AND Teugels, J. L. (1995). Ruin estimates under interest force. Insurance Math. Econom. 16, 7-22.

TANG, Q. (2004). The ruin probability of a discrete time risk model under constant interest rate with heavy tails. Scand. Actuarial J. 2004, 229-240.

TANG, Q. (2005). Asymptotic ruin probabilities of the renewal model with constant interest force and regular variation. Scand. Actuarial J. 2005, 1-5.

TANG, Q. AND TsitsiashVILI, G. (2003). Randomly weighted sums of subexponential random variables with application to ruin theory. Extremes 6, 171-188. 